\title{
Polymorph count for predicting non-gonococcal urethral infection: a model using Chlamydia trachomatis diagnosed by ligase chain reaction
}

\author{
L J Haddow, A Bunn, A J Copas, R Gilson, M Prince, G L Ridgway, S T Sadiq
}

Sex Transm Infect 2004;80:198-200. doi: 10.1136/sti.2003.006924

Background/objectives: The criteria for the diagnosis of non-gonococcal urethritis (NGU) on a Gram stained urethral smear are derived from previous studies which used culture as a diagnostic test for Chlamydia trachomatis. Our objectives were (1) to re-assess the relation between urethral polymorph count and $C$ trachomatis infection, using ligase chain reaction (LCR) as the diagnostic test; and (2) to assess other possible predictors of $C$ trachomatis infection such as symptoms, signs, demographic and behavioural variables. Methods: We collected data from 363 men consecutively attending a genitourinary medicine clinic (excluding those with gonorrhoea and follow up visits) who had a urethral smear and a urethral LCR test for $C$ trachomatis. The sensitivity and specificity of a discrete cut off in urethral polymorphonuclear leucocyte (PMNL) count as a diagnostic test for chlamydia urethritis were calculated. The associations between other variables, such as age and symptoms, and this infection were also estimated.

Results: $8 \%$ of men had $C$ trachomatis infection and $26 \%$ of men had a PMNL count of 5 or more. Of those men with chlamydia $37 \%$ did not have NGU; $20 \%$ of men with NGU had chlamydia. Adjusted odds ratios for risk of chlamydial infection were significant for age less than 30 relative to 40 years and over ladj OR 13.6; 95\% confidence interval 1.69 to 110$)$, a PMNL count of 20 or more $(6.56 ; 2.15$ to 20.0), a PMNL count of $5-19(3.59 ; 1.41$ to 9.15$)$, and the symptom of dysuria (3.27; 1.32 to 8.08 ). However a PMNL count of 5 or more was only $63 \%$ sensitive and $77 \%$ specific for $C$ trachomatis infection. No association between sexual behaviour and chlamydial infection was found in this setting. Conclusions: The PMNL count is associated with presence of chlamydial infection but a large proportion of men with chlamydia have PMNL counts below the recommended cut off for a diagnosis of NSU. Lower age and the presence of symptoms may be as predictive as the urethral polymorph count for chlamydial urethritis and possibly for other urethral infections.

$\mathrm{N}$ on-specific or non-gonococcal urethritis (NGU) is a frequent cause of symptoms in men and may be associated with pelvic inflammatory disease (PID) in female sexual contacts. ${ }^{1}$ The diagnosis in men is made by the absence of Gram negative intracellular cocci on a urethral smear and the presence of five or more polymorphonuclear leucocytes (PMNL) per high power field (hpf), averaged over five fields, and/or the presence of 10 or more PMNL per hpf on a Gram stained preparation from a first pass urine specimen. ${ }^{2}$ The diagnostic threshold is derived from studies showing an association with the clinical syndrome of urethritis. Previous work used culture as a diagnostic test for Chlamydia trachomatis and appeared to demonstrate an association between this organism and the presence of urethritis as defined by microscopy. ${ }^{3-6}$ Newer nucleic acid amplification tests (NAATs) have a higher sensitivity than culture (90-98\% compared to $60-80 \%) .{ }^{7}$ It is pertinent to reevaluate the relations between urethral infection, their clinical presentation, and PMNL counts from urethral smears.

The objective of the study was to determine the relation between $C$ trachomatis infection in men and urethral polymorph count by collecting data using ligase chain reaction (LCR) as the diagnostic test. The current recommended cut off in the polymorph count was to be assessed. We aimed to look at other predictors of $C$ trachomatis infection using routinely collected clinical data such as sexual behaviour, symptoms, and signs.

\section{METHODS}

Data were collected on all men having STI screens during a 4 week period in 2001. Regardless of symptoms, all subjects had urethral swabs for Gram stained urethral smear and LCR testing. Men who had concomitant gonococcal urethritis were excluded. From the Gram stains the average number of PMNL per hpf $(\times 1000$ magnification $)$ in the five fields of greatest density was calculated. A standard kit (LCx, Abbott Laboratories, Chicago, IL, USA ${ }^{7}$ ) was used for detection of $C$ trachomatis. All LCR results were retested for confirmation. Further information was retrieved from standardised proformas in the case notes including symptoms, sexual history, and examination findings. Self reported discharge and its presence on examination were recorded as two separate variables.

To analyse possible factors associated with chlamydia, the $\chi^{2}$ test was used for categorical variables and the MannWhitney test for continuous variables. Spearman rank correlation was used to assess the relation between time since last passing urine and urethral polymorph count, and interactions between these two variables and the LCR result were tested. Logistic regression was used to calculate adjusted odds ratios for those factors significantly associated at univariate analysis.

\section{RESULTS}

After excluding 19 men with gonococcal urethritis, 363 men were studied. The study population had a median age of 31.9 years (range 18-76), 82.0\% (228/278) were white, and $50 \%(182 / 363)$ were men who have sex with men.

Abbreviations: LCR, ligase chain reaction; NAATs, nucleic acid amplification tests; NGU, non-gonococcal urethritis; PID, pelvic inflammatory disease; PMNL, polymorphonuclear leucocytes 


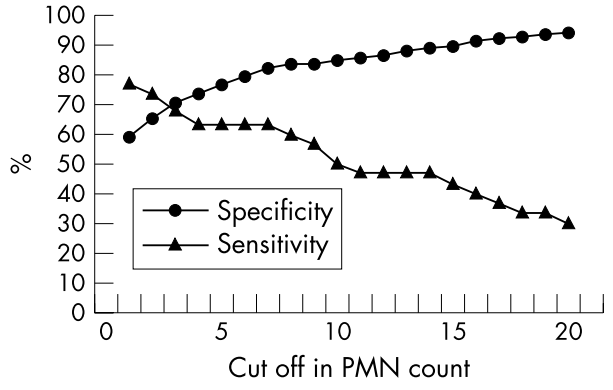

Figure 1 Each point on the $x$ axis represents a different possible cut off in polymorph count. Against this are plotted the resulting sensitivity and specificity of each cut off if it were a test for $C$ trachomatis infection. For example, a cut off of 10 means that all smears with 10 or more PMN per $\mathrm{hpf}$ are treated as chlamydia urethritis. For the purposes of this analysis, PMN counts recorded by the microscopist as "less than 5 " were plotted as if the actual value was 2 (includes two chlamydia positive and nine chlamydia negative results).

Sixty one of $363(16.8 \%)$ men presented with dysuria and $36 / 363(9.9 \%)$ complained of discharge. The prevalence of NGU diagnosed on urethral smear, was $26.4 \%$ (96/363) and the prevalence of $C$ trachomatis was $8.3 \%$ (30/363). Prevalence of $C$ trachomatis was greater at higher urethral polymorph counts-4.1\% in men with less than 5 PMNL per hpf compared to $31.0 \%$ in men with 20 or more PMNL per hpf $(\mathrm{p}<0.001)$.

Figure 1 displays the specificity and sensitivity of the urethral smear as if it were a test for chlamydia. More than one third of cases of chlamydia (11/30) had counts of less than 5 PMNL per hpf. Hence the diagnosis by a urethral PMNL count of 5 or more has a sensitivity of $63.3 \%$ for urethral chlamydia, and the specificity is $76.9 \%$. In our sample the positive predictive value of 5 or more PMNL per hpf for chlamydia was only $19.8 \%$, although the negative predictive value was $95.9 \%$. The point at which the lines cross is at 3 PMNL per hpf, defining a cut off where the specificity and sensitivity are balanced.

Table 1 summarises the associations between selected variables and $C$ trachomatis infection, presented as unadjusted and adjusted odd ratios. On multivariate analysis, the strongest associations with chlamydia infection were age, dysuria, and PMNL per hpf, although confidence intervals were wide. Discharge was significantly associated with higher PMNL count $(\mathrm{p}<0.001$, data not shown $)$ and was associated with chlamydia infection on univariate analysis but not multivariate analysis. No significant two way interactions were found between age, number of PMNL per hpf, or dysuria.

Other variables not tabulated which were not significantly associated with chlamydial infection included ethnicity, oral intercourse or unprotected vaginal or anal intercourse in the same time period, HIV status, recent use of antibiotics, sexual orientation, and number of sexual partners in the past 3 months. There was some evidence of higher rates in men who had had unprotected, insertive anal intercourse within the past 3 months $(p=0.075$, data not shown). In our sample $47 \%$ (160/341) of men had had two or more sexual partners in the past 3 months, and 48\% (165/343) had had a new partner in the same time period. We were unable to detect an effect on the association between number of PMNL per hpf and infection by the time since the patient last passed urine.

\section{DISCUSSION}

We found that increasing urethral polymorph count was associated with chlamydial infection in men. Although the overall prevalence of symptoms and disease was low, our study reflects current advice to screen all men attending GU clinics for NSU regardless of symptoms. Many men (in our sample 37\%) with chlamydial infection diagnosed on LCR have no microscopic evidence of urethritis (accepting that our study could have been improved by the use of microscopy on first pass urine samples). This finding may be partly the result of the high interobserver and intraobserver

Table 1 Frequency of $C$ trachomatis infection in association with selected variables

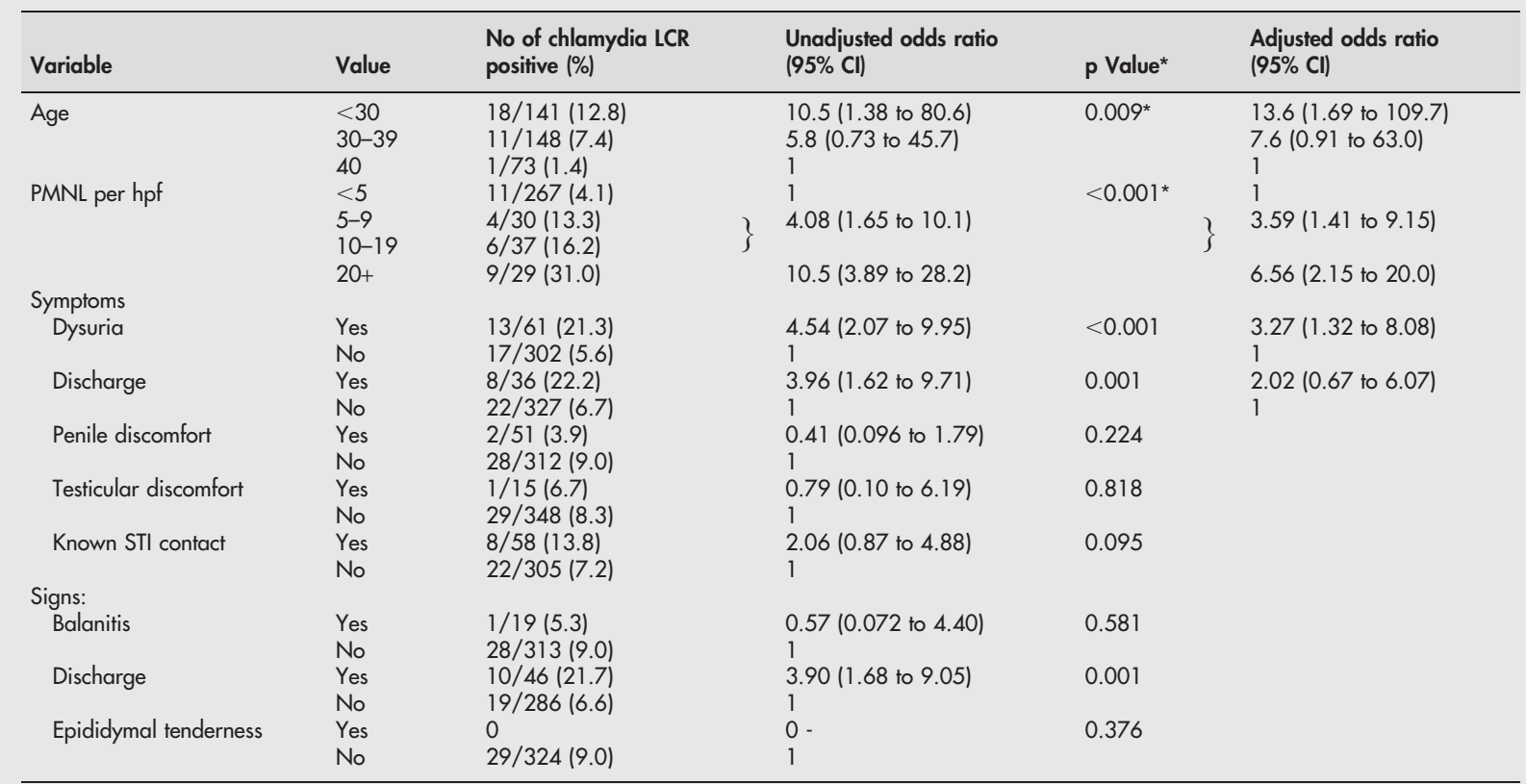

${ }^{*} \mathrm{p}$ Values listed refer to the $\chi^{2}$ test, except for variables asterisked which were analysed using the wilcoxon rank sum test. Multivariate analysis included variables found to be statistically significant on these tests. 
variation associated with microscopy, ${ }^{8}$ particularly at low PMNL counts, and questions the utility of a cut off in diagnosing urethral infection. Furthermore, our analysis showed symptoms of dysuria and even age to be about as strongly associated with chlamydia infection as urethral polymorph count, while the presence of discharge had no additional predictive power once a history of dysuria was elicited.

Despite the increasing availability of NAATs for chlamydia diagnosis, many questions regarding non-chlamydia NSU and asymptomatic urethritis are unanswered. Urethritis has been associated with Trichomonas vaginalis, ${ }^{9}$ although results were inconclusive when the diagnosis was made by PCR, and Mycoplasma genitalium ${ }^{10}{ }^{11}$ (which has also been associated with PID), but routine sensitive testing for these infections in men is generally unavailable. Microscopy still has a central diagnostic role in sexual health clinics and clinicians are advised to treat all men with NGU on the basis of this, supported by evidence of an association with pelvic inflammatory disease. ${ }^{1}$ Our data suggest that urethral polymorph count is not as strongly associated with $C$ trachomatis as previously assessed, and that age and symptoms should be incorporated into the predictive algorithm of urethral infection. Many clinicians adopt such an approach already. The epidemiology of chlamydia negative NGU may become easier to define as more sensitive tests for other organisms become available.

\section{ACKNOWLEDGEMENTS}

Additional data collection were provided by laboratory and nursing staff.

\section{CONTRIBUTORS}

All authors reviewed and commented on the manuscript; LH helped develop the study, collected data and co-wrote the paper and figures; $\mathrm{AB}$ developed the study, collected, and analysed data; $\mathrm{AC}$ performed statistical analysis and co-wrote relevant sections of the manuscript; RG developed the study and co-wrote the paper; MP helped develop the study and recorded laboratory data; STS conceived and developed the study and co-wrote the paper.
Authors' affiliations

L J Haddow, A Bunn, Department of Genitourinary Medicine, Camden Primary Care Trust, Mortimer Market Centre, London WCIE 6AU, UK A J Copas, R Gilson, S T Sadiq, Centre for Sexual Health and HIV Research, Department of Primary Care and Population Sciences, Royal Free and University College Medical School, University College London, London WCIE 6AU, UK

M Prince, G L Ridgway, Department of Clinical Microbiology, University College London Hospitals NHS Trust, London WC1E 6DB, UK

Correspondence to: Dr T Sadiq, The Courtyard Clinic, Department of Cellular and Molecular Medicine, St George's Hospital Medical School, Cranmer Terrace, London SW17 ORE, UK; s.sadiq@sghms.ac.uk

Accepted for publication 20 November 2003

\section{REFERENCES}

1 Kamwendo F, Johansson E, Moi H, et al. Gonorrhoea, genital chlamydial infection, and nonspecific urethritis in male partners of women hospitalised and treated for acute pelvic inflammatory disease. Sex Transm Dis 1993;20:143-6.

2 Clinical Effectiveness Group (AGUM and the MSSVD). National guideline on the management of non-gonococcal urethritis. Sex Transm Infect 1999;75(Suppl 1):S9-12, revised 2002 MSSVD.org.uk.

3 Swartz SL, Kraus SJ. Persistent urethral leukocytosis and asymptomatic chlamydial urethritis. J Infect Dis 1979;140:614-17.

4 Arya OP, Mallinson $\mathrm{H}$, Andrews $\mathrm{BE}$, et al. Diagnosis of urethritis: role of polymorphonuclear leukocyte counts in Gram-stained urethral smears. Sex Transm Dis 1984;11:10-17.

5 Hedin G, Abrahamsson G, Dahlberg E. Urethritis associated with Chlamydia trachomatis: comparison of leukocyte esterase dipstick test of first-voided urine and methylene blue-stained urethral smear as predictors of chlamydial infection. APMIS 2001;109:595-600.

6 Swartz SL, Kraus SJ, Herrmann KL, et al. Diagnosis and etiology of nongonococcal urethritis. J Infect Dis 1978;138:445-54.

7 Davies PO, Ridgway GL. The role of polymerase chain reaction and ligase chain reaction for the detection of Chlamydia trachomatis. Int J STD AIDS 1997;8:731-8.

8 Smith R, Copas AJ, Prince M, et al. Poor sensitivity and consistency of microscopy in the diagnosis of low-grade non-gonococcal urethritis. Sex Transm Infect 2003;79:487-90.

9 Wendel KA, Erbelding EJ, Gaydos CA, et al. Use of urine polymerase chain reaction to define the prevalence and clinical presentation of Trichomonas vaginalis in men attending an STD clinic. Sex Transm Infect 2003;79:151-3.

10 Horner PJ, Thomas B, Gilroy CB, et al. Do all men attending departments of genitourinary medicine need to be screened for non-gonococcal urethritis? Int J STD AIDS 2002;13:667-73.

11 Taylor-Robinson D, Horner PJ. The role of Mycoplasma genitalium in nongonococcal urethritis. Sex Transm Infect 2001;77:229-31. 